\title{
Qi-Deficiency Related Increases in Disease Susceptibility Are Potentially Mediated by the Intestinal Microbiota
}

\author{
Ke Ma $\left(D,{ }^{1}\right.$ Jieyu Chen, ${ }^{1}$ Liuyan Kuang, ${ }^{1}$ Jianlu Bi $\left(\mathbb{D},{ }^{2}\right.$ Jingru Cheng, ${ }^{3}$ Fei Li, ${ }^{4}$ Xiaomin Sun, \\ Xiaoli Nie, ${ }^{1}$ Yanyan Liu $\mathbb{D},{ }^{1}$ Ren Luo $\mathbb{D},{ }^{1}$ and Xiaoshan Zhao $\mathbb{D}^{1}$ \\ ${ }^{1}$ School of Traditional Chinese Medicine, Southern Medical University, Guangzhou, Guangdong 510515, China \\ ${ }^{2}$ Department of Endocrinology, Guangdong Second Traditional Chinese Medicine Hospital, Guangzhou 510095, China \\ ${ }^{3}$ Department of Nephrology, The First Affiliated Hospital of Zhengzhou University, Zhengzhou 450002, China \\ ${ }^{4}$ Department of Traditional Chinese Medicine, The Affiliated Ganzhou Hospital of Nanchang University, Ganzhou 314000, China
}

Correspondence should be addressed to Xiaoshan Zhao; zhaoxs0609@163.com

Received 14 July 2018; Accepted 2 October 2018; Published 23 October 2018

Academic Editor: Raffaele Capasso

Copyright (c) $2018 \mathrm{Ke}$ Ma et al. This is an open access article distributed under the Creative Commons Attribution License, which permits unrestricted use, distribution, and reproduction in any medium, provided the original work is properly cited.

\begin{abstract}
Qi-deficiency (QX) is thought to promote the body's susceptibility to disease, but the underlying mechanism through which this occurs is not clear. We surveyed the traditional Chinese medicine constitution (TCMC) of healthy college students to identify those that were PH (balanced TCMC constitution) and QX (unbalanced TCMC constitution). We then used high-throughput sequencing of the 16SrRNA V3-4 region in fecal microbiota samples to identify differences between those obtained from PH and QX individuals. Our results demonstrated that the alpha diversity of QX samples was significantly lower than that of PH samples $(p<0.05)$ and that beta diversity was remarkably different in QX and PH samples. Four and 122 bacterial taxa were significantly overrepresented in QX and PH groups, respectively. The genera Sphingobium, Clostridium, and Comamonas were enriched in the QX group and had a certain pathogenic role. The QX group also showed a statistically significant lack of probiotics and anti-inflammatory bacteria such as Bifidobacterium and Bdellovibrio. The functional potential of QX bacterial taxa was reduced in fatty acid metabolism and butanoate metabolism. We contend that the imbalanced intestinal microbiota in QX and the following functional changes in metabolism influence immunity and energy metabolism, which could increase susceptibility to disease.
\end{abstract}

\section{Introduction}

Traditional Chinese medicine constitution (TCMC) refers to relatively stable physical and psychological characteristics and is based on epidemiological investigations [1], clinical manifestations [2], and genomic research among Han Chinese [3, 4]. There is one balanced constitution of TCMC, $\mathrm{PH}$, and eight unbalanced constitutions, including Qi-deficiency (QX) [5]. TCMC theory digitizes empirical Traditional Chinese Medicine diagnosis and treatment and provides a standardized guideline which is particularly useful for clinics [6]. Previous findings show that QX is an early stage in cancer [7, 8], heart disease [9], hypertension [10], diabetes [11], chronic fatigue syndrome [12, 13], and depression [14] and that $\mathrm{PH}$ is a protective factor against these conditions $[9,15]$.
$\mathrm{PH}$ people are energetic and fit, are not susceptible to illness, and have stable and powerful pulses and quality sleep. QX people are characterized by fatigue, are lacking in strength, and have weak pulses. QX is related to overwork, working pressure, and aging [16]. QX could promote the body's susceptibility to disease, but its mechanism is not clear.

Studies have shown the downregulation of immunerelated genes including those involved in major histocompatibility complex [17] and interleukin- $1 \beta$ binding [18] in QX people. Our previous studies revealed the presence of metabolic disorders in the plasma of healthy QX people, including the presence of betaine and alanine [19]. Previous reports indicated that betaine in plasma positively correlates with Clostridium in feces [20] and that Lactobacillus casei Shirota could improve plasma alanine-aminotransferase levels [21]. These cases revealed that the specific plasma metabolites 
TABLE 1: General situation.

\begin{tabular}{lccc}
\hline characteristics & QX $(\mathrm{n}=19)$ & PH $(\mathrm{n}=16)$ & $p$ value \\
\hline Age (years) & $20.75 \pm 1.47$ & $20.13 \pm 1.31$ & 0.2007 \\
Sex $($ male /female) & $7 / 12$ & $5 / 11$ & 0.728 \\
BMI $\left(\mathrm{kg} / \mathrm{m}^{2}\right)$ & $20.18 \pm 3.31$ & $20.94 \pm 1.31$ & 0.3951 \\
QX transformed score & $48.58 \pm 9.56$ & $10.94 \pm 5.82$ & - \\
Health Status Scores & $79.23 \pm 5.32$ & $81.47 \pm 7.15$ & 0.2962 \\
\hline
\end{tabular}

No statistical significance between the two groups in age, sex, BMI, and health status scores $(p>0.05)$.

of QX were associated with intestinal microbiota. However, visceral manifestation of QX has also indicated spleenand lung-deficiencies and some results indicate that these deficiencies induce compositional and functional changes in intestinal microbiota $[22,23]$.

We hypothesize that QX-related increases in disease susceptibility are potentially mediated by intestinal microbiota. We performed pyrosequencing of the 16SrRNA gene in healthy QX and PH Han Chinese college students, to compare their intestinal microbiota, and predicted metabolic function to explore the biological mechanism of QX.

\section{Method}

2.1. Study Subjects. This study is reviewed and approved by China Ethics Committee of Registering Clinical Trials (No. ChiECRCT-20170064) and Chinese Clinical Trials Registry (No. ChiCTR-IOR-17012986). We screened 461 healthy PH students and 166 QX students from 5987 college students of southern Han ethnicity (Figure 1). The inclusion criteria were as follows: first, unremarkable physical and blood tests including urea, electrolytes, and liver function tests; second, healthy status according to the results of Subhealth Measurement Scale V1.0 [24]; third, results of PH/QX in Chinese Constitution Medicine Questionnaire [25, 26]. Random sampling was used to select $21 \mathrm{PH}$ and $21 \mathrm{QX}$ students for 16SrRNA sequencing (V3-4). These 42 students were prohibited to take antibiotics, probiotics, or Chinese herbal medicines for 16 weeks. We obtained $16 \mathrm{PH}$ and $19 \mathrm{QX}$ sequencing datasets.

2.2. Fecal Sample Collection and Sequencing. Fresh fecal samples were collected in sterile container, kept at $4^{\circ} \mathrm{C}(<0.5$ $\mathrm{h})$, and then frozen at $-80^{\circ} \mathrm{C}$. According to the manufacturer's instructions, total fecal DNA was extracted using DNA Stool mini kit (Shenzhen Bioeasy Biotechnologies, Co., Ltd.). We amplified the V3-4 region of the 16SrRNA gene by PCR using the universal primers $\mathrm{V} 4 \mathrm{~F}$ and $\mathrm{V} 4 \mathrm{R}$ (Table S1). $25 \mu$ l reaction mixture was $0.25 \mu \mathrm{l} 5 \mathrm{U} / \mu \mathrm{l}$ Premix Taq, $1 \mu \mathrm{l}$ template DNA, $0.5 \mu \mathrm{l} 10 \mu \mathrm{M}$ barcodes forward primer, $0.5 \mu \mathrm{l} 10 \mu \mathrm{M}$ reverse primer, and $16.75 \mu \mathrm{l}$ double-distilled $\mathrm{H}_{2} \mathrm{O}$. The PCR cycle conditions were an initial denaturation at $94^{\circ} \mathrm{C}$ for $2 \mathrm{~min}$, $30 \mathrm{cycles}$ of $94^{\circ} \mathrm{C}$ for $30 \mathrm{~s}, 52^{\circ} \mathrm{C}$ for $30 \mathrm{~s}$, and $72^{\circ} \mathrm{C}$ for 40 $\mathrm{s}$, and a final extension at $72^{\circ} \mathrm{C}$ for $5 \mathrm{~min}$. PCR products were sequenced using Illumina MiSeq instrument (PE 300, Illumina, San Diego, California, USA) at Guangzhou RiboBio Co., Ltd.
Sequencing reads were clustered by Illumina paired barcoded-sequencing (end) (BIPES) (PE) process for preliminary analysis; the rest of the sequence was screened by UCHIME and the suspected chimeric sequence was removed. The two-stage clustering (TSC) was used for extracting the OTU, with sequences having greater than or equal to $97 \%$ similarity being assigned to the same OTU. Alpha diversity was performed with Chaol, observed species, Shannon and Simpson. Beta diversity was performed with principle coordinate analysis (PCoA) based on UniFrac distance [27]. The linear discriminant analysis (LDA) with effect size measurements (LefSe) [28] was used to identify statistically significant different bacterial species in QX group and $\mathrm{PH}$ group. PICRUSt [29] was used to predict the metabolic function of the 16SrRNA gene datasets with KEGG Orthologs classification ( $p<0.05$, Welch's t-test) [30].

2.3. Statistical Analysis. Epidata 3.0 was used to establish a database. Enumeration data was tested by chi-square. quantitative data was tested by independent-samples t-test. Kruskal-Wallis, PCoA, LEfSe, and PICRUSt analyzed the sequencing data. Statistical analyses were performed with the SPSS 20.0 (SPSS Inc., Chicago, IL, USA). P < 0.05 was considered to be of statistical significance.

\section{Results}

3.1. Students and Samples. There was no statistical significance between the two groups in age, sex, BMI, and health status scores (Table $1, p>0.05$ ). We obtained 119,909 sequences from 19 QX samples and 218,480 sequences from $16 \mathrm{PH}$ samples (Table S2).

3.2. Microbiota in $Q X$ Were Different from $P H$. The numbers of observed species, Shannon and Simpson indices, were significantly lower in the QX group than in the $\mathrm{PH}$ group $(p<0.05$, Figure 2). PCoA (UniFrac) demonstrated a clear clustering of the microbial populations of the QX group, which were separated from the $\mathrm{PH}$ group (Figure 3).

3.3. Phylum, Family, and Genus Level Taxonomic Distribution of Intestinal Microbiota in QX Populations. Bacteroidetes and Firmicutes were the top two phyla identified in QX samples and accounted for $47.64 \%$ and $39.23 \%$ of the total valid reads, respectively. In $\mathrm{PH}$ samples, Firmicutes and Bacteroidetes accounted for $43.99 \%$ and $38.52 \%$ (Figure $4(\mathrm{a})$ ). At the family level, based on the average relative abundance, nine species 


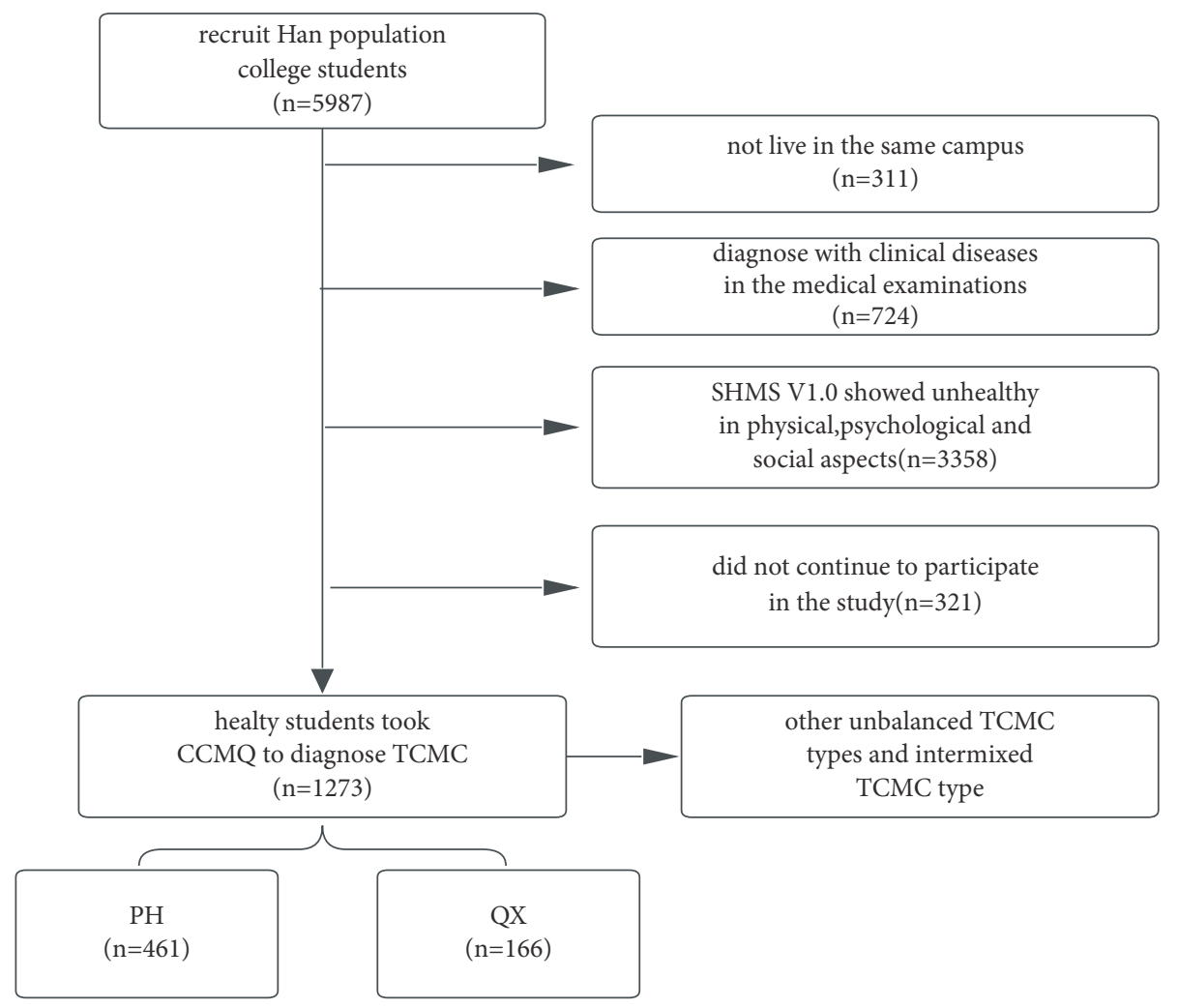

FIgURE 1: The sequence flow diagram of inclusion criteria for PH samples and QX samples.
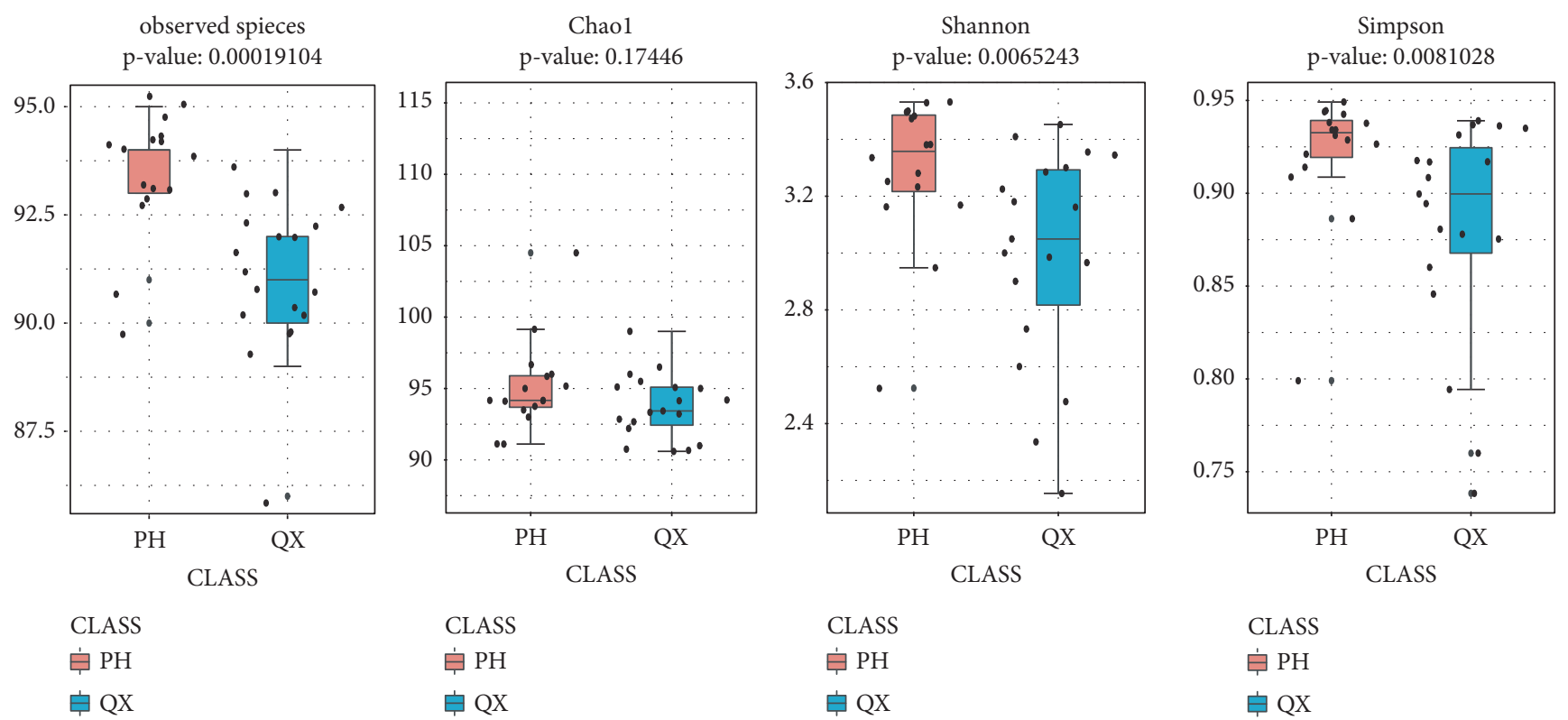

FIgURE 2: Bacterial alpha diversity in QX (blue) and PH (red) populations. Observed species, Shannon and Simpson indices demonstrate that the diversity in QX people is significantly lower than that in $\mathrm{PH}$ people. Mann-Whitney, $p<0.01$.

were dominant $(\geq 1 \%)$ in $\mathrm{PH}$ samples, and 10 species were dominant in QX samples. Bacteroidaceae and Alcaligenaceae were enriched in QX, whereas Ruminococcaceae, Fusobacteriaceae, and Bifidobacteriaceae were enriched in PH (Figure $4(\mathrm{~b}))$. At the genera level, 12 genera were dominant in $\mathrm{PH}$, and 11 genera were dominant in QX. Bacteroides, Megamonas, Lachnospira, and Sutterella were enriched in QX, and Ruminococcus, Fusobacterium, Megasphaera, and Coprococcus were more abundant in $\mathrm{PH}$ (Figure 4(c)). $\mathrm{PH}$ contained more Ruminococcus and Coprococcus which can 


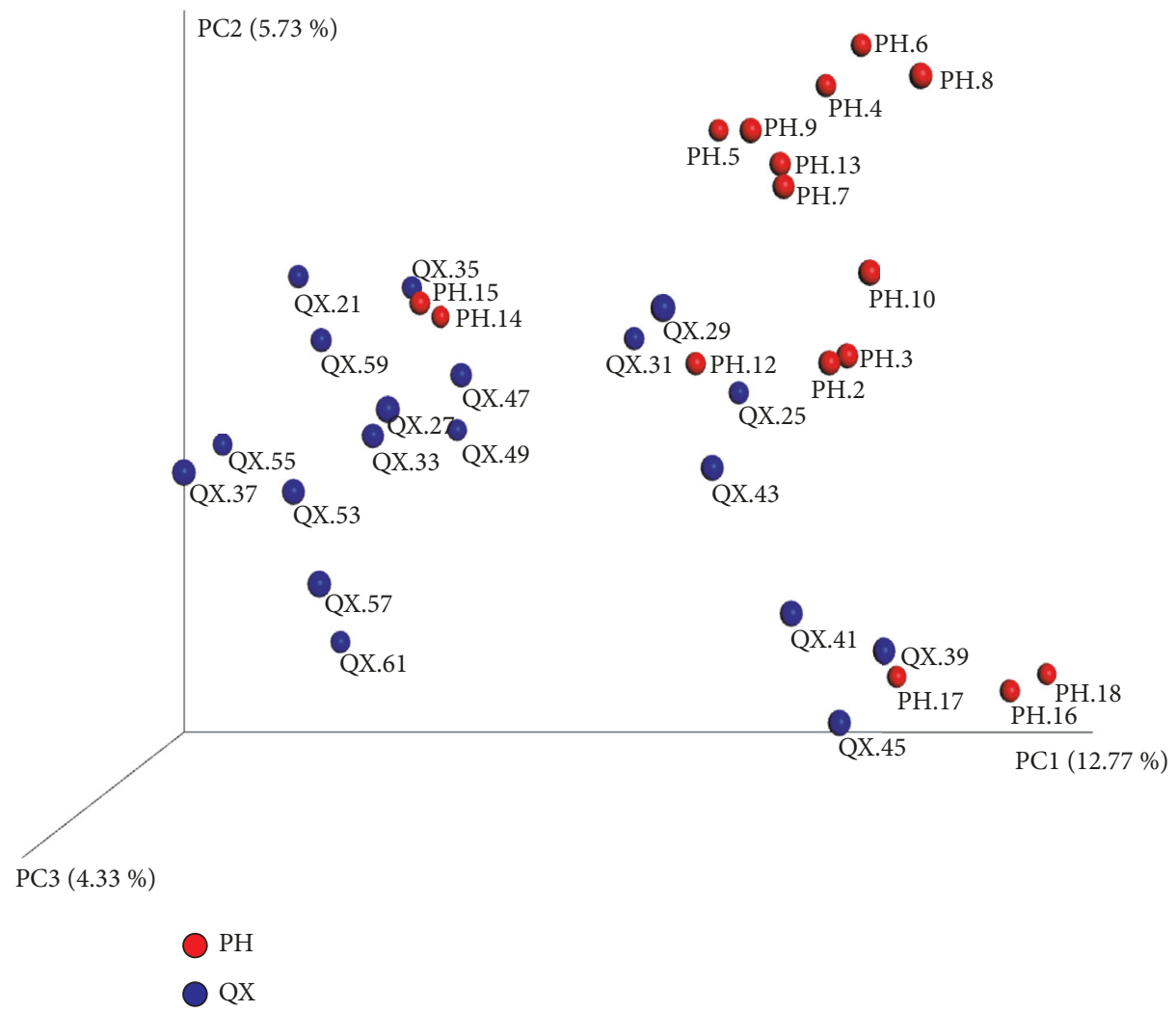

FIgURE 3: PCoA (unweighted) of intestinal microbiota in QX (blue) and PH (red) populations. Principal coordinate axis 1 (12.99\% variability) and principal coordinate axis 2 (5.68\% variability) highlight a clear clustering.

produce butyrate [31], whereas Sutterella, present in QX samples, could give rise to unbalanced microflora by degrading secretory IgA and IgA itself [32].

3.4. Decreased Bacteria Associated with Short-Chain Fatty Acid Production and Butanoate Metabolism Are Prominent Features of QX. LefSe showed biomarkers for QX and $\mathrm{PH}$ $(\mathrm{LDA}>2, p<0.05)$. Four and 122 taxa were enriched in $\mathrm{QX}$ and $\mathrm{PH}$ samples, respectively (Figures 5(a) and 5(b)). At the genus level, Sphingobium, Clostridium, and Comamonas were significantly overrepresented in QX and Megasphaera, Veillonella, Veillonella parvula, Bifidobacterium, Blautia, Streptococcus, Bdellovibrio, and Paraprevotella were significantly overrepresented in PH. Moreover, at the family level, Veillonellaceae, Bifidobacteriaceae, Pseudomonadaceae, Lactobacillales, Lachnospiraceae, Streptococcaceae, and Bdellovibrionaceae were significantly overrepresented in $\mathrm{PH}$.

Of the biomarkers for QX, Comamonas is a cellulolytic microbe [33] and Sphingobium [34] and Clostridium [35, 36] are associated with inflammation. Of the biomarkers PH for, Megasphaera [37], Veillonellaceae [38], Pseudomonadaceae [39], Streptococcaceae [40], and Bifidobacterium $[41,42]$ can produce and metabolize fat, starches, protein, and polysaccharides, which are significantly lacking in QX. $\mathrm{PH}$ biomarkers Bifidobacterium and lactobacilli can ferment inulin-type fructans to cross-feed the microbiota and significantly stimulate the production of n-butyrate $[43,44]$.
Additionally, $\mathrm{PH}$ samples were rich in anti-inflammatory bacteria, including Bifidobacterium [45, 46], Streptococcus [47], Lachnospiraceae [48], and Bdellovibrio [49]. Metabolites of Bifidobacterium, including acetic acid, lactic acid, ethanol, formic acid, vitamins, and amino acids, can also stimulate the immune response and enhance the function of NK cells and the proliferation of T lymphocytes[50, 51]. Bdellovibrio, considered as potential antibiotic substitutes, could also increase Coprococcus, which is reduced in irritable bowel syndrome and influences microbial balance [52].

PICRUSt was used to investigate the potential function and metabolic pathways of the taxa, based on Kyoto Encyclopedia of Genes and Genomes (KEGG) modules. Of note, QX had a lower proportion of bacterial genes related to fatty acid metabolism and butanoate metabolism and a higher proportion of bacterial genes related to amino and nucleotide sugar metabolism and RNA degradation (Figure 6, $p<0.05$, Welch's inverted CI method effect size $>0.01$ ).

\section{Discussion}

Here, we described our investigation of intestinal microbiota characteristics among healthy QX and $\mathrm{PH}$ Han college students in Southern China. We performed 16SrRNA gene sequencing to examine the different microbiota in QX and $\mathrm{PH}$ groups and to explain the biological mechanism underlying susceptibility to disease in QX individuals. Our results 

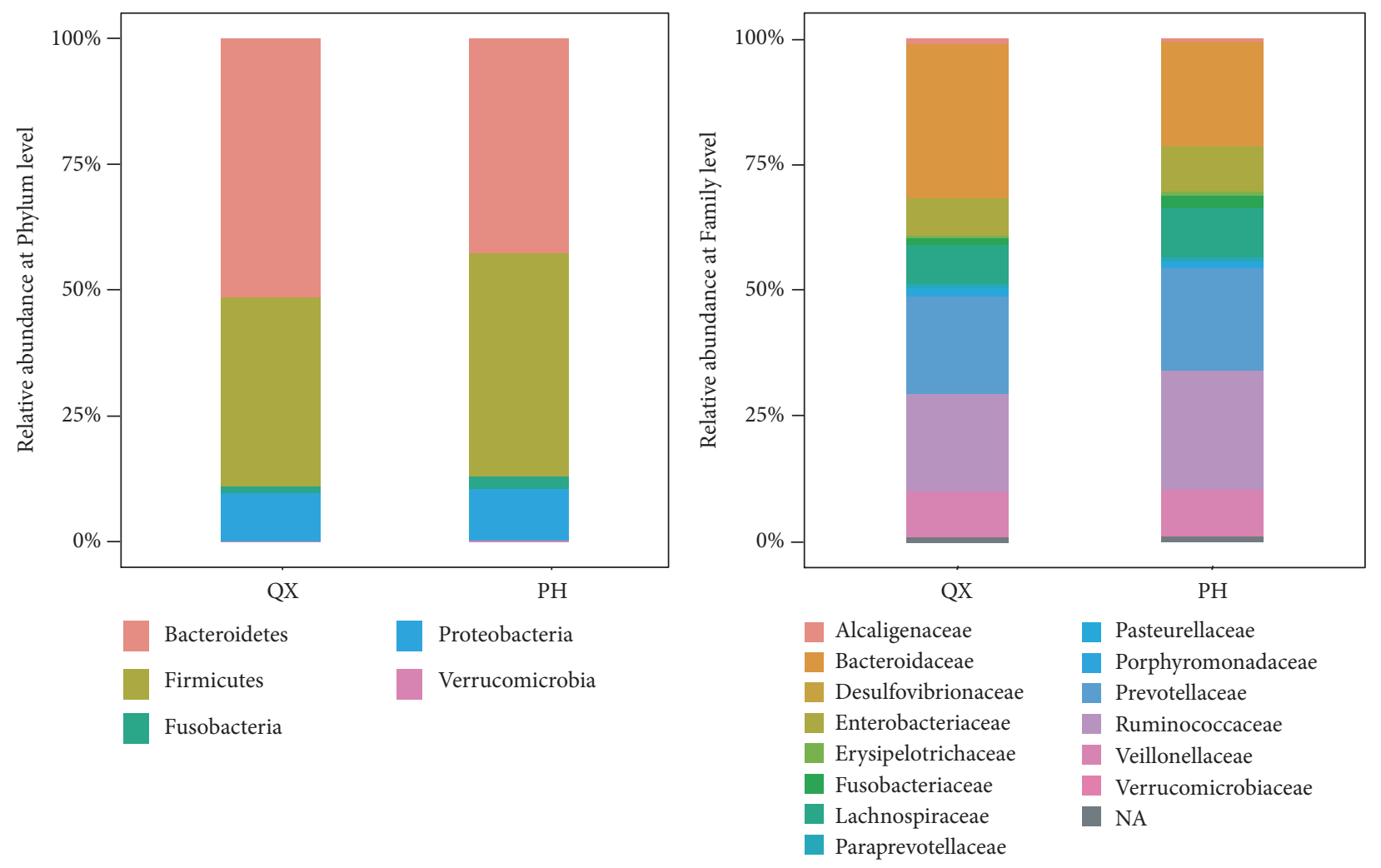

(a)

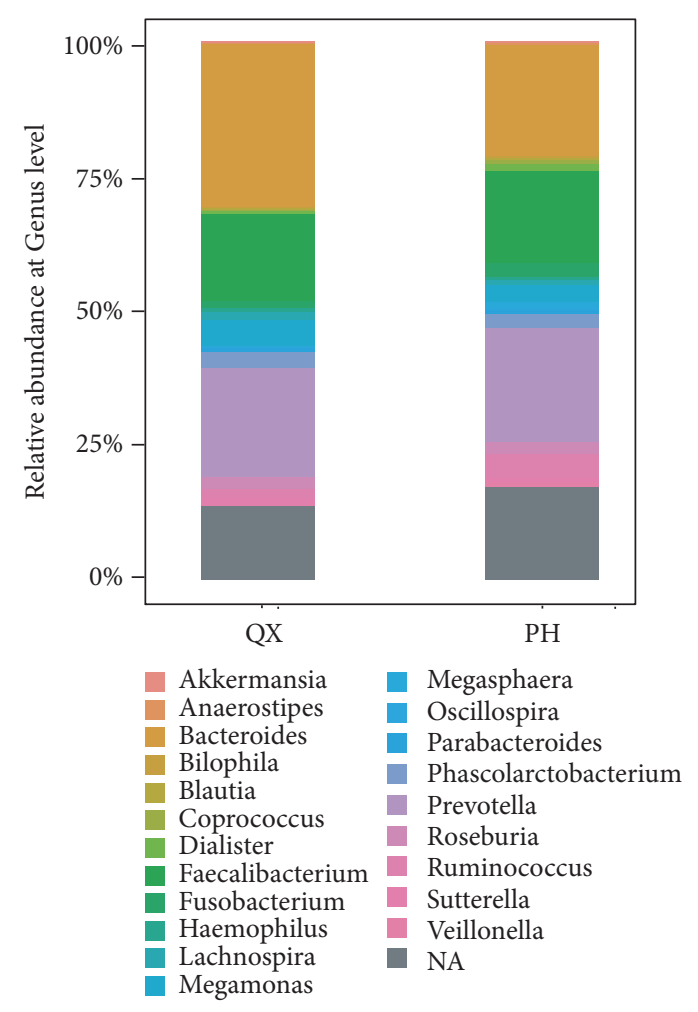

$(\mathrm{c} 1)$

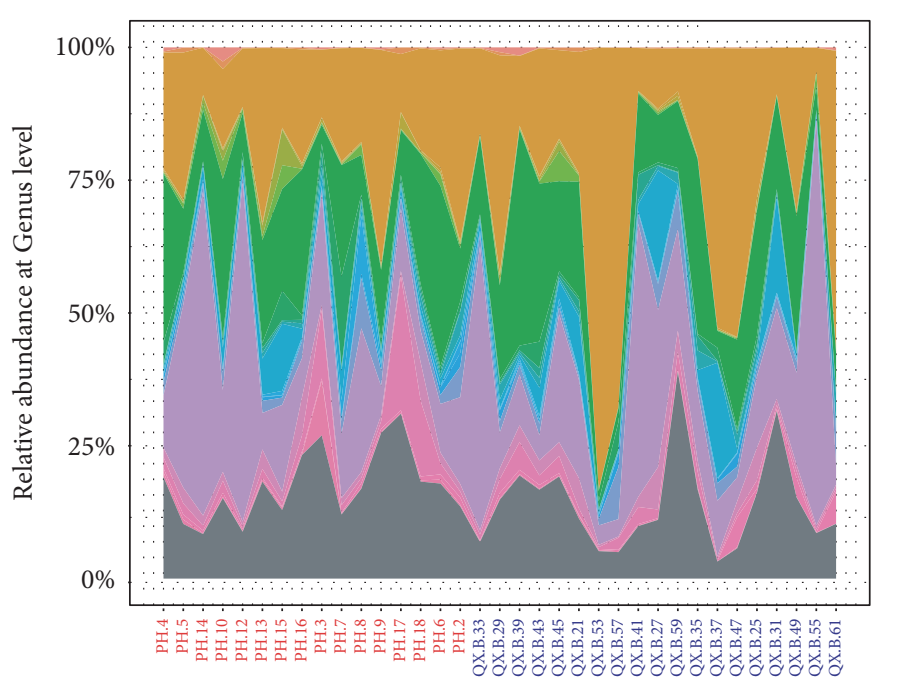

(c)

FIgURE 4: Relative abundance of intestinal microbiota at phylum (a), family (b), and genus (c) levels in PH and QX populations based on 16SrRNA sequencing. (b) shows that the taxa median relative abundance at the family level is $\geq 1 \%$ of total abundance. (c) shows that the taxa relative abundance at the genus level is $\geq 1 \%$ of the total abundance within groups (c1) and individuals (c2). 

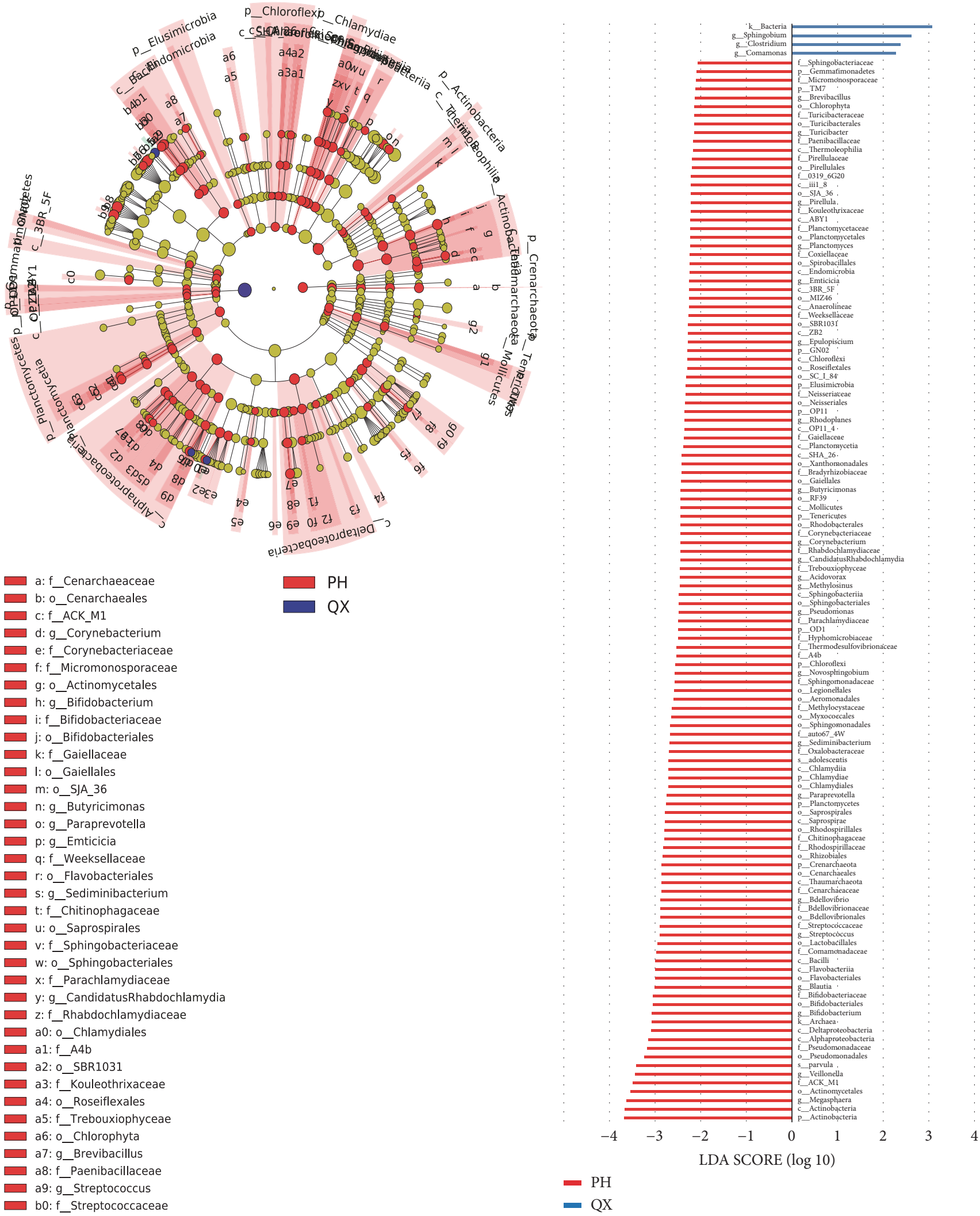

(a)

(b)

FIGURE 5: LefSe shows significantly discriminative features in QX (blue) and PH (red) populations. LDA $>2.0, \mathrm{p}<0.05$. (a) Cladograms of the hierarchical structure. (b) Differential abundance bacterial taxa between the two groups presented as a histogram. The key microbiota taxa in QX were genus Sphingobium, Clostridium, and Comamonas. 


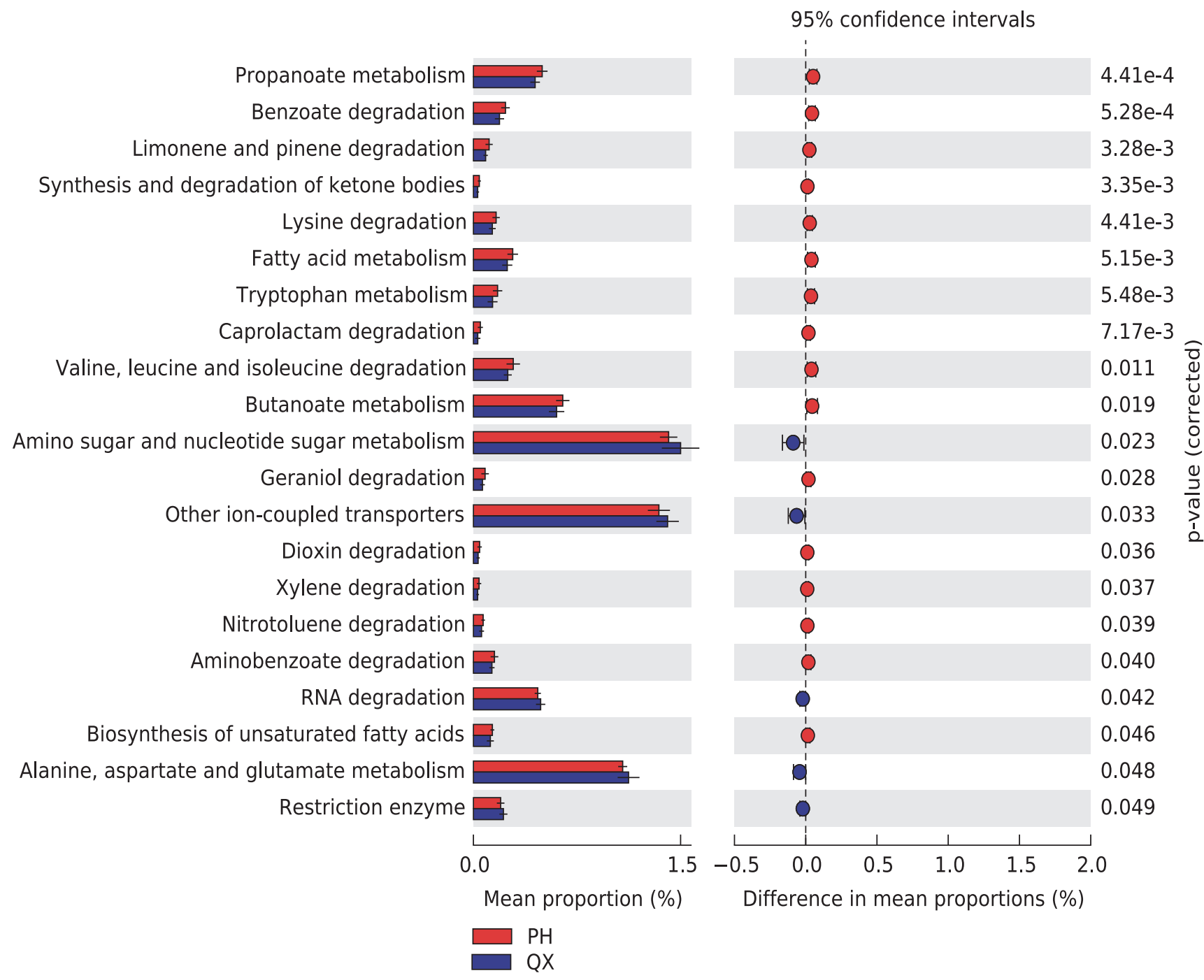

FIGURE 6: PICRUSt shows that the 21 predicted KOs significantly differ in their distribution in healthy QX (blue) and PH (red) populations. Functional modules involved in amino and nucleotide sugar metabolism and in RNA degradation are more abundant in QX than they are in PH. P $<0.05$, Welch's t-test, Welch's inverted CI method, and effect size $>0.01$.

show that the bacterial taxa data of healthy people were similar to that of Asian populations reported in previous studies $[53,54]$ but that the microbiota of QX group was statistically different from that of the $\mathrm{PH}$ group.

Functionally, QX microbiota have relatively decreased fatty acid and butanoate metabolism potentials and relatively enriched carbohydrate metabolism (amino sugar and nucleotide sugar metabolism) and RNA degradation potentials. The imbalance of energy metabolism caused by alterations in intestinal microbiota is associated with QX-related symptoms. QX people demonstrate significantly higher BMI scores and waist-to-hip ratios and significantly lower scores in back muscle strength [55]. QX symptoms include a reduction of resting metabolic rate, which results in flabby muscles, vulnerability to fatigue, feeble pulse, shallow breathing [56], and potential concern with disturbances in energy metabolism. Research has shown that GLUT4, a critical protein in glucose metabolism, increases following low-intensity exercise [57] and that moderate-intensity exercise increases fatty acid oxidation to adapt energy metabolism [58]. Per mole of oxygen consumed, the oxidation of carbohydrates produces $15 \%$ more ATP than the oxidation of lipids does [59]. However, lipid oxidation produces greater energy density compared to carbohydrate oxidation, and fatty acids can go straight to the muscles' mitochondria for energy. Therefore, we postulate that QX and $\mathrm{PH}$ people adopt different optimal-fuel strategies to acclimatize themselves to daily energy expenditure. Compared with $\mathrm{PH}$ people, QX people tend to adopt a carbohydrate-based metabolism to prolong the time for which energy is maintained, which makes it possible to induce the symptoms of lower metabolic rate. $\mathrm{PH}$ people tend to adopt fatty acid metabolism and butanoate metabolism. Lipid oxidation has a greater energy density than does carbohydrate oxidation, which efficiently elevates thermogenesis capacity. Higher rates of fat oxidation generally reflect well-trained body conditions, while lower 
fat oxidation rates may be related to obesity and insulin resistance [60].

The microbiota identified in the QX group had reduced levels of probiotics and decreased anti-inflammatory bacteria than did those identified in the $\mathrm{PH}$ group. The alteration of intestinal microbiota and unbalanced metabolism in QX, including reduction of fatty acid and butanoate metabolism, can increase disease susceptibility. Short-chain fatty acids (SCFAs), especially butyrate, play an important role in the regulation of host immunity. SCFAs directly contact intestinal epithelium cells (IECs), and SCFAs, especially butyrate, are used as ATP sources for energy metabolism. However, SCFAs also enhance IEC immune surveillance by increasing the expression of certain antimicrobial peptides and maintaining the integrity of intestinal mucosa [61-63]. For example, commensal microbe-derived butyrate induced the differentiation of colonic regulatory $\mathrm{T}$ cells, which have a central role in the suppression of inflammatory and allergic responses [64]. Furthermore, butyrate and valproic acid upregulated B cell microRNAs in human and mouse B cells to modulate antibody and autoantibody responses [65].

In conclusion, this study demonstrates that intestinal microbiota in the QX group showed lower biological diversity and possessed a different microbial signature than did that of the PH group. We contend that the QX's imbalanced intestinal microbiota and concomitant functional changes in metabolism potentially influence immunity and energy metabolism, which could increase susceptibility to disease.

\section{Data Availability}

The data used to support the findings of this study are available from the corresponding author upon request.

\section{Conflicts of Interest}

The authors declare that there are no conflicts of interest.

\section{Authors' Contributions}

Xiaoshan Zhao and Ren Luo designed the experiments. Ke Ma, Jieyu Chen, Liuyan Kuang, Jianlu Bi, Jingru Cheng, Fei $\mathrm{Li}$, and Xiaoli Nie performed the experiments. Jieyu Chen and Ke Ma analyzed the data. Ke Ma, Liuyan Kuang, and Xiaoshan Zhao prepared the manuscript. Xiaoshan Zhao and Xiaomin Sun were responsible for study supervision. All authors were involved in the formulation of the research questions. Ke Ma, Jieyu Chen, and Liuyan Kuang contributed equally to this work

\section{Acknowledgments}

The authors thank their study participants. This work was supported by the Key Project of National Natural Science Foundation of China (no. 81830117), the National Science Foundation of China (no. 81673840, no. 81703891, no. 81760821, and no. 81703952), Natural Science Foundation of Guangdong Province, China (no. 2017A030313791 and no. 2016A030310311), the Science \& Technical Plan of Guangzhou, Guangdong, China (no. 2014Y2-00504), and TCM State Administration Foundation of Guangdong Province, China (20164001).

\section{Supplementary Materials}

Table S1. The information of primers. Table S2. Samples sequencing and alpha diversity of the intestinal microbiota. (Supplemental Materials)

\section{References}

[1] W. Qi and Z. Yan-bo, "Epidemiological investigation of constitutional types of Chinese medicine in general population: Base on 21, 948 epidemiological investigation data of nine provinces in China," Journal of Traditional Chinese Medicine, p. 12, 2009.

[2] H. Yumin, W. Li, S. Fengting, C. Gengwu, Z. Dafeng, and X. Yun, "Clustering study of TCM constitution," Chinese Journal of Basic Medicine in traditional chinese medicine, p. 10, 1996.

[3] L. Wang Ji and W. Bai Ming-Hua, "Genomics study on nine types of TCM constitution," China Journal of Traditional Chinese Medicine and Pharmacy, pp. 3871-3873, 2014.

[4] R. Yu, D. Liu, Y. Yang et al., "Expression profiling-based clustering of healthy subjects recapitulates classifications defined by clinical observation in Chinese medicine," Journal of Genetics and Genomics, vol. 44, no. 4, pp. 191-197, 2017.

[5] W. Qi, "The Classification of Nine Kinds of Basic TCM Constitution and the Basis of Diagnosis," Journal of Beijing University of Traditional Chinese Medicine, pp. 1-8, 2005.

[6] J. Wang, Y. Li, C. Ni, H. Zhang, L. Li, and Q. Wang, "Cognition research and constitutional classification in Chinese medicine," American Journal of Chinese Medicine, vol. 39, no. 4, pp. 651660, 2011.

[7] C. A. Xuedong, A Preliminary Study on the Pathogenesis Underlying Nasopharyngeal Precancerous Lesion Induced by the Interactive Effeets of Qi-dificient Physique Status with $N, \mathrm{~N}^{\text {- }}$ dinitrosopiperazine [Doctoral Dissertation]: HuNan university of traditional chinese medicine [Doctoral, thesis], HuNan university of traditional chinese medicine, 2005.

[8] F. Tao, P. Lü, C. Xu et al., "Metabolomics Analysis for Defining Serum Biochemical Markers in Colorectal Cancer Patients with Qi Deficiency Syndrome or Yin Deficiency Syndrome," Evidence-Based Complementary and Alternative Medicine, vol. 2017, 2017.

[9] Y. Zhu, H. Shi, Q. Wang, Y. Wang, X. Yu, J. Di et al., Association between Nine Types of TCM Constitution and Five Chronic Diseases: A Correspondence Analysis Based on a Sample of 2,660 Participants, vol. 2017, Evid Based Complement Alternat Med, 2017.

[10] Y.-B. Zhu, Q. Wang, Q.-W. Deng, J. Cai, X.-H. Song, and X. Yan, "Relationships between constitutional types of traditional Chinese medicine and hypertension," Journal of Chinese Integrative Medicine, vol. 8, no. 1, pp. 40-45, 2010.

[11] Y. Juan, Traditional Chinese medical body constitution, its related clinical characteristics, and diabetes-associated chronic complications in patients with type 2 diabetes, DaLian Medical Universit, 2015.

[12] L. Xinping, "Correlations between TCM Constitution and Chronic Fatigue Syndrome," World Chinese Medicine, pp. 11711174, 2017. 
[13] H. Yan, The correlation of chronic fatigue symdrome and its impact factors and TCM constitution and clinical research [Doctoral Dissertation], GuangZhou university of chinese medicion, 2013.

[14] Y.-C. Liao, C.-Y. Chou, C.-T. Chang et al., "Qi deficiency is associated with depression in chronic hemodialysis patients," Complementary Therapies in Medicine, vol. 30, pp. 102-106, 2017.

[15] T. Wang, J. Chen, X. Sun et al., "Effects of TCMC on transformation of good health status to suboptimal health status: a nested case-control study," Evidence-Based Complementary and Alternative Medicine, vol. 2015, Article ID 259727, 8 pages, 2015.

[16] H. C. Chiang, S. T. Yang, K. C. Lee, P. Y. Huang, M. Hsu, and H. H. Chang, "From theory to clinic: key components of qi deficiency in traditional Chinese medicine," Altern Ther Health Med, vol. 18, pp. 28-36, 2012.

[17] W. Ruilin, Preliminary Research on Gene Expression Profile of Peripheral Blood of the constitution of Qi deficiency [Master Dissertation]: BeiJing university of chinese medicine [Master, thesis], Preliminary Research on Gene Expression Profile of Peripheral Blood of the constitution of Qi deficiency [Master Dissertation], BeiJing university of chinese medicine, 2005.

[18] Z. Xiaojun, T. Daofa, W. Shizhen, and R. Yan, “Genital Stable and Gene Expression with High Gastritis Cancer Family in Qi Deficiency. Information on Traditional Chinese Medicine," Genital Stable and Gene Expression with High Gastritis Cancer Family in Qi Deficiency. Information on Traditional Chinese Medicine, pp. 59-62, 2009.

[19] B. Jianlu, C. Jieyu, C. Jingru, and Y. Bingyan, “The comparative plasma metabonomic analysis of qi-deficiency constitution and gentleness constitution," Journal of Tropical Medicine, pp. 717720, 2014.

[20] A. B. Ross, S. J. Bruce, A. Blondel-Lubrano et al., "A whole-grain cereal-rich diet increases plasma betaine, and tends to decrease total and LDL-cholesterol compared with a refined-grain diet in healthy subjects," British Journal of Nutrition, vol. 105, no. 10, pp. 1492-1502, 2011.

[21] S. Wagnerberger, A. Spruss, G. Kanuri et al., "Lactobacillus casei Shirota protects from fructose-induced liver steatosis: a mouse model," The Journal of Nutritional Biochemistry, vol. 24, no. 3, pp. 531-538, 2013.

[22] P. Y. Z. S. Liu Jia, "Investigation on intestinal microflora of elderly patients with spleen deficiency by $16 \mathrm{~S}$ rDNA DGGE analysis," Journal of Traditional Chinese Medicine and Pharmacy, pp. 1566-1569, 2010.

[23] K. Yanhua, Structural Modulation of Gut Microbiota And Mechanism Research in Rats with Chronic Obstructive Pulmonary Disease Treated with Recuperating Lung Decoction, BeiJing university of chinese medicine, 2016.

[24] F. Liyi, X. Jun, L. Ren, Q Jincai, and Z. Jinhua, "Analysis and Build-up for Sub-Health Evaluating Indicat or System," chinese general practice, pp. 37-40, 2011.

[25] China Association of Traditional Chinese Medicine and Pharmacy, "Classification and Judgment of TCM Constitution (ZYYXH/T157-2009)," World Journal of Integrated Traditional and Western Medicine, pp. 303-304, 2009.

[26] W. Qi, Z. Yanbo, X. Hesheng, and L. Shao, "primary compiling of constitution in chinese medicine questionnaire," chinese journal of clinical rehabilitation, p. 12, 2006.

[27] C. Lozupone, M. Hamady, and R. Knight, "UniFrac-an online tool for comparing microbial community diversity in a phylogenetic context," BMC Bioinformatics, vol. 7, article 371, 2006.
[28] N. Segata, J. Izard, L. Waldron et al., "Metagenomic biomarker discovery and explanation," Genome Biology, vol. 12, no. 6, article R60, 2011.

[29] M. G. I. Langille, J. Zaneveld, J. G. Caporaso et al., "Predictive functional profiling of microbial communities using $16 \mathrm{~S}$ rRNA marker gene sequences," Nature Biotechnology, vol. 31, no. 9, pp. 814-821, 2013.

[30] M. Kanehisa, S. Goto, Y. Sato, M. Kawashima, M. Furumichi, and M. Tanabe, "Data, information, knowledge and principle: back to metabolism in KEGG," Nucleic Acids Research, vol. 42, no. 1, pp. D199-D205, 2014.

[31] S. E. Pryde, S. H. Duncan, G. L. Hold, C. S. Stewart, and H. J. Flint, "The microbiology of butyrate formation in the human colon," FEMS Microbiology Letters, vol. 217, no. 2, pp. 133-139, 2002.

[32] C. Moon, M. T. Baldridge, M. A. Wallace, C.-A. D. Burnham, H. W. Virgin, and T. S. Stappenbeck, "Vertically transmitted faecal IgA levels determine extra-chromosomal phenotypic variation," Nature, vol. 521, no. 7550, pp. 90-93, 2015.

[33] P. Kanokratana, S. Wongwilaiwalin, W. Mhuantong, S. Tangphatsornruang, L. Eurwilaichitr, and V. Champreda, "Characterization of cellulolytic microbial consortium enriched on Napier grass using metagenomic approaches," Journal of Bioscience and Bioengineering, 2017.

[34] S. Chen, C. Tsai, Y. Lee et al., "Intestinal microbiome in children with severe and complicated acute viral gastroenteritis," Scientific Reports, vol. 7, no. 1, 2017.

[35] S. Arase, Y. Watanabe, H. Setoyama, N. Nagaoka, M. Kawai, and S. Matsumoto, "Disturbance in the mucosa-associated commensal bacteria is associated with the exacerbation of chronic colitis by repeated psychological stress; is that the new target of probiotics?" PLoS ONE, vol. 11, no. 8, 2016.

[36] J. De La Cuesta-Zuluaga, N. T. Mueller, V. Corrales-Agudelo et al., "Metformin is associated with higher relative abundance of mucin-degrading akkermansia muciniphila and several shortchain fatty acid-producing microbiota in the gut," Diabetes Care, vol. 40, no. 1, pp. 54-62, 2017.

[37] J. L. Rychlik, R. La Vera, and J. B. Russell, "Amino acid deamination by ruminal Megasphaera elsdenii strains," Current Microbiology, vol. 45, no. 5, pp. 340-345, 2002.

[38] M. J. Bonder, E. F. Tigchelaar, X. Cai et al., "The influence of a short-term gluten-free diet on the human gut microbiome," Genome Medicine, vol. 8, no. 1, 2016.

[39] Y. Liu, J. Li, J. Yu et al., "Disorder of gut amino acids metabolism during CKD progression is related with gut microbiota dysbiosis and metagenome change," Journal of Pharmaceutical and Biomedical Analysis, vol. 149, pp. 425-435, 2018.

[40] K. Murphy, D. Curley, T. F. O'Callaghan et al., “The Composition of Human Milk and Infant Faecal Microbiota Over the First Three Months of Life: A Pilot Study," Scientific Reports, vol. 7, no. 1, 2017.

[41] C. Milani, G. Andrea Lugli, S. Duranti et al., "Bifidobacteria exhibit social behavior through carbohydrate resource sharing in the gut," Scientific Reports, vol. 5, 2015.

[42] C. Milani, F. Turroni, S. Duranti et al., "Genomics of the genus Bifidobacterium reveals species-specific adaptation to the glycan-rich gut environment," Applied and Environmental Microbiology, vol. 82, no. 4, pp. 980-991, 2016.

[43] L. De Vuyst and F. Leroy, "Cross-feeding between bifidobacteria and butyrate-producing colon bacteria explains bifdobacterial competitiveness, butyrate production, and gas production," 
International Journal of Food Microbiology, vol. 149, no. 1, pp. 73-80, 2011.

[44] T. Tsukahara, K. Hashizume, H. Koyama, and K. Ushida, "Stimulation of butyrate production through the metabolic interaction among lactic acid bacteria, Lactobacillus acidophilus, and lactic acid-utilizing bacteria, Megasphaera elsdenii, in porcine cecal digesta," Animal Science Journal, vol. 77, no. 4, pp. 454-461, 2006.

[45] P. Markowiak and K. Ślizewska, "Effects of probiotics, prebiotics, and synbiotics on human health," Nutrients, vol. 9, no. 9, article no. 1021, 2017.

[46] Q. Zeng, X. He, S. Puthiyakunnon et al., "Probiotic mixture golden Bifido prevents neonatal Escherichia coli K1 translocation via enhancing intestinal defense," Frontiers in Microbiology, vol. 8, 2017.

[47] M. Wlodarska, C. Luo, R. Kolde et al., "Indoleacrylic Acid Produced by Commensal Peptostreptococcus Species Suppresses Inflammation," Cell Host \& Microbe, vol. 22, no. 1, pp. 25-37.e6, 2017.

[48] M. Saresella, L. Mendozzi, V. Rossi et al., "Immunological and clinical effect of diet modulation of the gut microbiome in multiple sclerosis patients: A pilot study," Frontiers in Immunology, vol. 8, 2017.

[49] K. Shatzkes, C. Tang, E. Singleton et al., "Effect of predatory bacteria on the gut bacterial microbiota in rats," Scientific Reports, vol. 7, 2017.

[50] H. Wang, J. Gong, W. Wang et al., "Are there any different effects of Bifidobacterium, Lactobacillus and Streptococcus on intestinal sensation, barrier function and intestinal immunity in PI-IBS mouse model?" PLoS ONE, vol. 9, no. 3, 2014.

[51] K. M. Maslowski and C. R. MacKay, "Diet, gut microbiota and immune responses," Nature Immunology, vol. 12, no. 1, pp. 5-9, 2011.

[52] E. Malinen, L. Krogius-Kurikka, A. Lyra et al., "Association of symptoms with gastrointestinal microbiota in irritable bowel syndrome," World Journal of Gastroenterology, vol. 16, no. 36, pp. 4532-4540, 2010.

[53] J. Zhang, Z. Guo, Z. Xue et al., "A phylo-functional core of gut microbiota in healthy young Chinese cohorts across lifestyles, geography and ethnicities," The ISME Journal, vol. 9, no. 9, pp. 1979-1990, 2015.

[54] Y. Nam, M. Jung, S. W. Roh, M. Kim, J. Bae, and E. Dias-Neto, "Comparative Analysis of Korean Human Gut Microbiota by Barcoded Pyrosequencing," PLoS ONE, vol. 6, no. 7, p. e22109, 2011.

[55] D. Meng, Research of Characteristics of Shape and Function in Undergraduate Students with Pardal Constitution and Exercise Intervention to the People with Qi-insumciency Constitution [Doctoral Dissertation], ShanDong university of chinese medicien, 2008.

[56] S. Mun, S. Kim, K.-H. Bae, and S. Lee, "Cold and Spleen-Qi Deficiency Patterns in Korean Medicine Are Associated with Low Resting Metabolic Rate," Evidence-Based Complementary and Alternative Medicine, vol. 2017, Article ID 9532073, 2017.

[57] W. Zhen, The effect of different intensity of treadmill exercise on mitochondrial glucose and fatty acid oxidation key factors in rats myocytes [Master Dissertation], HeBei Medical University, 2016.

[58] D. P. Koonen, C. R. Benton, Y. Arumugam et al., "Different mechanisms can alter fatty acid transport when muscle contractile activity is chronically altered," American Journal of Physiology-Endocrinology and Metabolism, vol. 286, no. 6, pp. E1042-E1049, 2004.
[59] M. Brand, "The efficiency and plasticity of mitochondrial energy transduction," Biochemical Society Transactions, vol. 33, no. 5, pp. 897-904, 2005.

[60] R. K. Randell, I. Rollo, T. J. Roberts, K. J. Dalrymple, A. E. Jeukendrup, and J. M. Carter, "Maximal Fat Oxidation Rates in an Athletic Population," Medicine \& Science in Sports \& Exercise, vol. 49, no. 1, pp. 133-140, 2017.

[61] C. Iraporda, A. Errea, D. E. Romanin et al., "Lactate and short chain fatty acids produced by microbial fermentation downregulate proinflammatory responses in intestinal epithelial cells and myeloid cells," Immunobiology, vol. 220, no. 10, pp. 11611169, 2015.

[62] L. Macia, J. Tan, A. T. Vieira et al., "Metabolite-sensing receptors GPR43 and GPR109A facilitate dietary fibre-induced gut homeostasis through regulation of the inflammasome," Nature Communications, vol. 6, article 7734, 2015.

[63] M. Y. Lin, M. R. De Zoete, J. P. M. Van Putten, and K. Strijbis, "Redirection of epithelial immune responses by short-chain fatty acids through inhibition of histone deacetylases," Frontiers in Immunology, vol. 6, 2015.

[64] Y. Furusawa, Y. Obata, S. Fukuda et al., "Commensal microbederived butyrate induces the differentiation of colonic regulatory T cells," Nature, vol. 504, no. 7480, pp. 446-450, 2013.

[65] C. A. White, E. J. Pone, T. Lam et al., "Histone deacetylase inhibitors upregulate B cell microRNAs that silence AID and blimp-1 expression for epigenetic modulation of antibody and autoantibody responses," The Journal of Immunology, vol. 193, no. 12, pp. 5933-5950, 2014. 


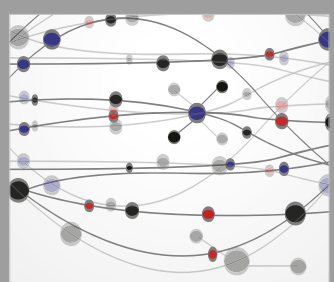

The Scientific World Journal
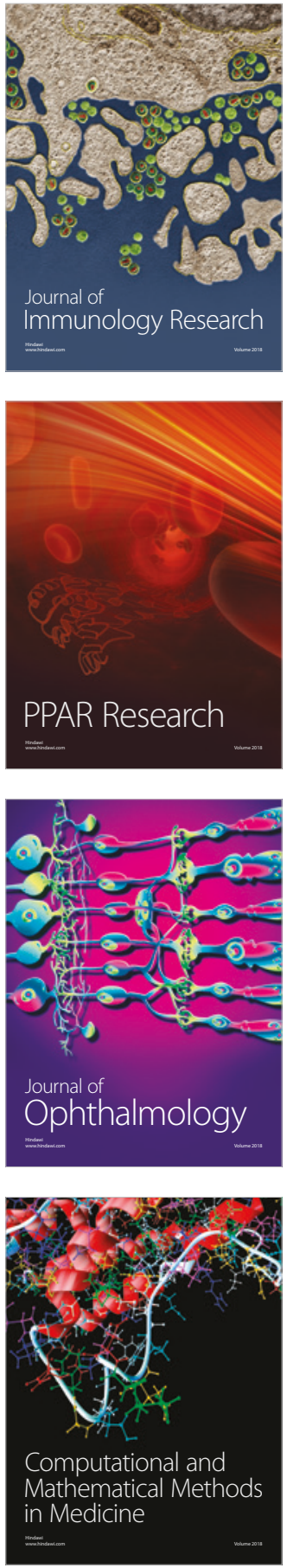

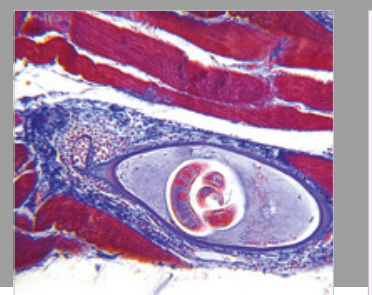

Gastroenterology Research and Practice

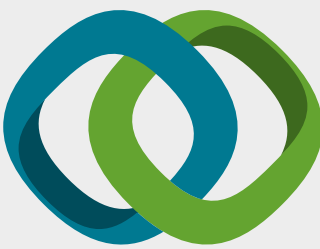

\section{Hindawi}

Submit your manuscripts at

www.hindawi.com
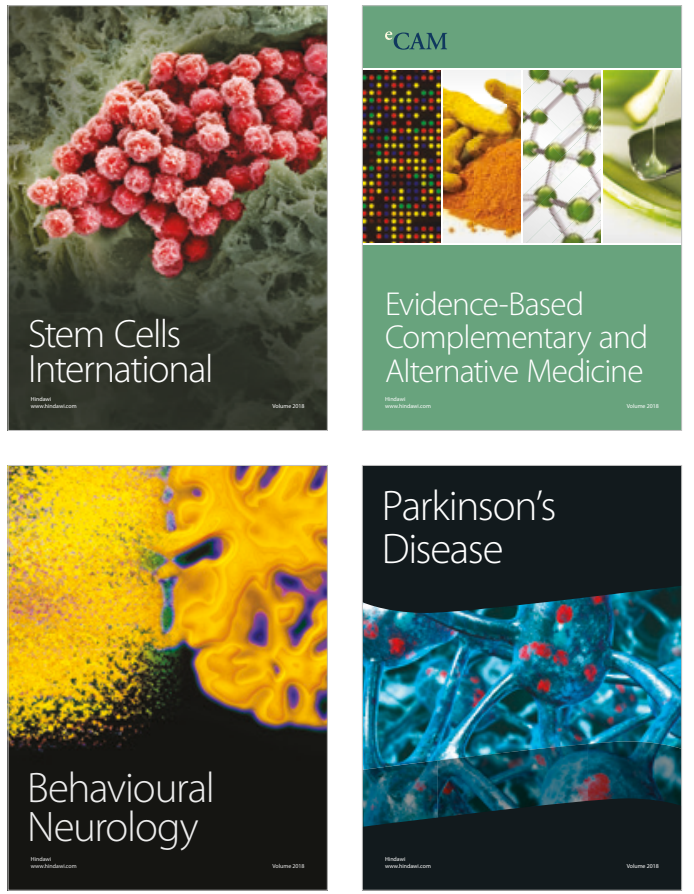

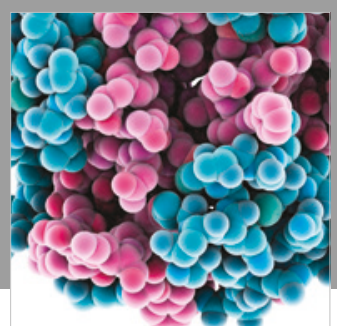

ournal of

Diabetes Research

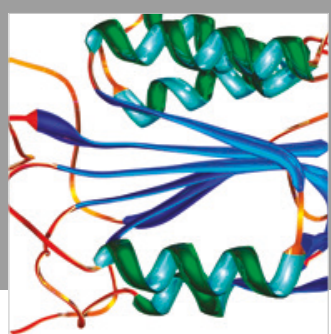

Disease Markers
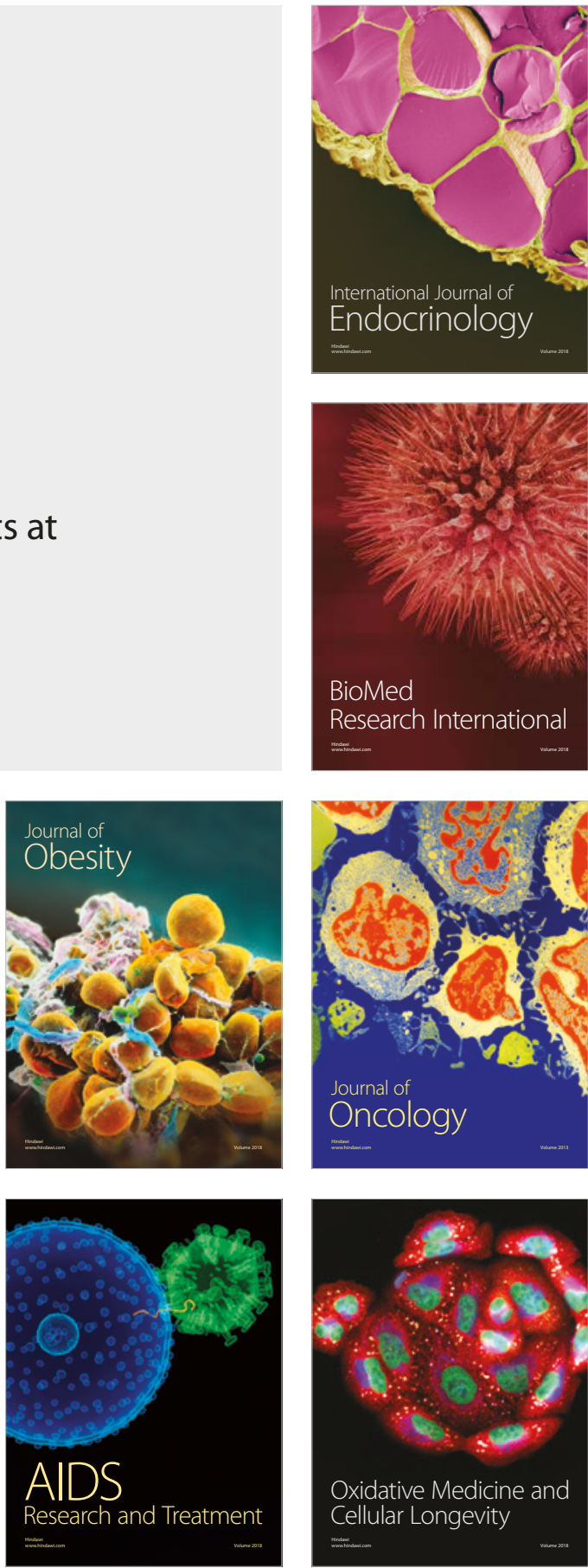